\title{
ESTRUCTURA Y LÍMITES DE LA PONDERACIÓN
}

\author{
Carlos Bernal Pulido* \\ Universidad Externado de Colombia
}

\section{Introducción}

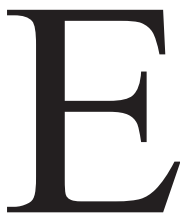

xisten dos formas básicas para aplicar normas: la ponderación y la subsunción. Las reglas se aplican mediante la subsunción, al paso que la ponderación es la manera de aplicar los principios. Es por ello que la ponderación se ha convertido en un criterio metodológico indispensable para el ejercicio de la función jurisdiccional, especialmente la que se desarrolla en los Tribunales Constitucionales, que se encargan de la aplicación de normas que, como los derechos fundamentales, tienen la estructura de principios. A pesar de ello, la ponderación se sitúa en el centro de muchas discusiones teóricas, que revelan que algunos aspectos tales como su estructura y sus límites, aun distan de estar del todo claros. El objetivo de esta ponencia es analizar estos problemas. Con todo, de antemano es preciso aclarar el concepto de ponderación ${ }^{1}$.

\section{El concepto de ponderación}

La ponderación es la forma en que se aplican los principios jurídicos, es decir, las normas que tienen la estructura de mandatos de optimización. Estas normas no determinan exactamente lo que debe hacerse, sino que ordenan "que algo sea realizado en la mayor medida posible, dentro de las posibilidades jurídicas y reales existentes." ${ }^{2}$ Las posibilidades jurídicas

* Profesor de filosofía del derecho y derecho constitucional de la Universidad Externado de Colombia (Bogotá)

${ }^{1}$ Para un análisis detenido del concepto de ponderación: Cfr. Carlos Bernal Pulido, El principio de proporcionalidad y los derechos fundamentales, Centro de Estudios Políticos y Constitucionales, Madrid, 2003, p. 757 y ss.

${ }^{2}$ Cfr. Robert Alexy, Teoría de los derechos fundamentales, Traducción de Ernesto Garzón Valdés, Centro de Estudios Políticos y Constitucionales, Madrid, 1997, p. 86 y 87. 
están determinadas por los principios y reglas opuestas, y las posibilidades reales se derivan de enunciados fácticos.

Para establecer esa "mayor medida posible" en que debe realizarse un principio, es necesario confrontarlo con los principios opuestos o con los principios que respaldan a las reglas opuestas. Esto se lleva a cabo en una colisión entre principios. Existe una colisión entre principios, cuando en un caso concreto son relevantes dos o más disposiciones jurídicas, que fundamentan prima facie dos normas incompatibles entre sí, y que pueden ser propuestas como soluciones para el caso. Se presenta una colisión entre principios, por ejemplo, cuando los padres de una niña, que profesan el culto evangélico, y en razón del respeto a los mandamientos de esta doctrina religiosa, se niegan a llevarla al hospital, a pesar de que corre peligro de muerte $^{3}$. Si referimos este caso al derecho constitucional colombiano, observaremos que las disposiciones de los artículos 19 y 16 de la Constitución, que establecen, respectivamente, la libertad de cultos y el derecho al libre desarrollo de la personalidad $^{4}$, fundamentan un principio que en la mayor medida posible permite decidir a los padres, si de acuerdo con sus creencias deben llevar o no a sus hijos al hospital. Este principio entra en colisión con los principios del derecho a la vida y a la salud de la niña, establecidos por los artículos 11, 44 y 49 de la Constitución, que ordenan proteger la vida y la salud de los niños en la mayor medida posible ${ }^{5}$. La incompatibilidad normativa se presenta en este caso, porque, de los artículos 19 y 16 se deriva que está permitido prima facie a los padres de la niña, decidir si la llevan o no al hospital, mientras que de los artículos 11, 44 y 49 se sigue que llevar a la niña al hospital, es una conducta ordenada prima facie por los derechos fundamentales.

La ponderación es la forma de resolver esta incompatibilidad entre normas prima facie. Para tal fin, la ponderación no garantiza una articulación sistemática material de todos los principios jurídicos, que, habida cuenta de

${ }^{3}$ El ejemplo es de la sentencia T-411 de 1994 de la Corte Constitucional Colombiana.

${ }^{4}$ Art. 19 Const. Col.: "Se garantiza la libertad de cultos. Toda persona tiene derecho a profesar libremente su religión y a difundirla en forma individual o colectiva. Todas las confesiones religiosas e iglesias son igualmente libres ante la ley".

Art. 16 Const. Col.: "Todas las personas tienen derecho al libre desarrollo de su personalidad sin más limitaciones que las que imponen los derechos de los demás y el orden jurídico".

${ }^{5}$ Art. 11 Const. Col.: "El derecho a la vida es inviolable. No habrá pena de muerte".

Art. 44 Const. Col.: "Son derechos fundamentales de los niños: la vida, la integridad física, la salud, la seguridad social, la alimentación equilibrada... Los derechos de los niños prevalecen sobre los derechos de los demás".

Art. 48 Const. Col.: "La atención de la salud y el saneamiento ambiental son servicios públicos a cargo del Estado. Se garantiza a todas las personas el acceso a los servicios de promoción, protección y recuperación de la salud..." 
su jerarquía, resuelva de antemano todas las posibles colisiones entre ellos. Por el contrario, al igual que el silogismo, la ponderación es sólo una estructura, que está compuesta por tres elementos, mediante los cuales se puede fundamentar una relación de precedencia condicionada entre los principios en colisión ${ }^{6}$, para así establecer cuál de ellos debe determinar la solución del caso concreto.

\section{La estructura de la ponderación}

Quizá ha sido Robert Alexy quien con mayor claridad y precisión haya expuesto la estructura de la ponderación. De acuerdo con Alexy, para establecer la relación de precedencia condicionada entre los principios en colisión, es necesario tener en cuenta tres elementos que forman la estructura de la ponderación: la ley de ponderación, la fórmula del peso y las cargas de argumentación.

\section{La ley de la ponderación}

Según la ley de la ponderación, "Cuanto mayor es el grado de la no satisfacción o de afectación de uno de los principios, tanto mayor debe ser la importancia de la satisfacción del otro"7.

Si se sigue esta ley, la ponderación se puede dividir en tres pasos que el propio Alexy identifica claramente: "En el primer paso es preciso definir el grado de la no satisfacción o de afectación de uno de los principios. Luego, en un segundo paso, se define la importancia de la satisfacción del principio que juega en sentido contrario. Finalmente, en un tercer paso, debe definirse si la importancia de la satisfacción del principio contrario justifica la afectación o la no satisfacción del otro" .

Es pertinente observar que el primero y el segundo paso de la ponderación son análogos. En ambos casos, la operación consiste en establecer un grado de afectación o no satisfacción - del primer principio- y de importancia en la satisfacción - del segundo principio-. En adelante nos referiremos a ambos fenómenos como la determinación del grado de afectación de los principios en el caso concreto ${ }^{9}$. Alexy sostiene que el grado de afectación de

\footnotetext{
${ }^{6}$ Esto es lo que Alexy llama la ley de la colisión. Cfr. Teoría de los derechos fundamentales, op. cit., pp. 90 y ss.

${ }^{7}$ Ibídem, pp. 161 y ss.

${ }^{8}$ Cfr. Robert Alexy, "Epílogo a la Teoría de los derechos fundamentales", traducción de Carlos Bernal Pulido, REDC, núm. 66, 2002, pp. 32.

${ }^{9}$ En esta terminología puede decirse que mientras el primer principio se afecta de manera negativa, el segundo se afecta de forma positiva. Siguiendo la notación de Alexy, simbolizaremos el grado de afectación o no satisfacción del primer principio en el caso concreto como $I P i C$ y la importancia en la satisfacción del segundo principio, también en el caso concreto, como WPjC. Cfr. Ibídem, pp. 40 y ss.
} 
los principios puede determinarse mediante el uso de una escala tríadica o de tres intensidades. En esta escala, el grado de afectación de un principio en un caso concreto puede ser "leve", "medio" o "intenso". Así, por ejemplo, la afectación de la vida y la salud de la niña, que se originaría al permitir a los padres evangélicos no llevarla al hospital, podría catalogarse como intensa, dado el peligro de muerte. De forma correlativa, la satisfacción de la libertad de cultos de los padres, que se derivaría de dicha permisión, podría graduarse sólo como media o leve.

Conviene reconocer que el grado de afectación de los principios en el caso concreto no es la única variable relevante para determinar, en el tercer paso, si la satisfacción del segundo principio justifica la afectación del primero. La segunda variable es el llamado "peso abstracto" de los principios relevantes ${ }^{10}$. La variable del peso abstracto se funda en el reconocimiento de que, a pesar de que a veces los principios que entran en colisión tengan la misma jerarquía en razón de la fuente del derecho en que aparecen -por ejemplo, dos derechos fundamentales que están en la Constitución tienen la misma jerarquía normativa-, en ocasiones uno de ellos puede tener una mayor importancia en abstracto, de acuerdo con la concepción de los valores predominante en la sociedad. Así, por ejemplo, eventualmente puede reconocerse que el principio de protección a la vida tiene un peso abstracto mayor que la libertad, por cuanto para poder ejercer la libertad es necesario tener vida, o como sostiene Joseph Raz, porque la vida es un presupuesto para que podamos acceder a todas las cosas que tienen valor y ejercer todos nuestros derechos ${ }^{11}$. De la misma manera, la jurisprudencia constitucional de diversos países en ocasiones ha reconocido un peso abstracto mayor a la libertad de información frente al derecho al honor o a la intimidad, por su conexión con el principio democrático, o a la intimidad y a la integridad física y psicológica sobre otros principios, por su conexión con la dignidad humana $^{12}$.

A lo anterior se agrega una tercera variable, que denotaremos como la variable $S$. Ella se refiere a la seguridad de las apreciaciones empíricas, que versan sobre la afectación que la medida examinada en el caso concreto -por ejemplo, permitir que los padres evangélicos decidan si llevan o no a

${ }^{10}$ Siguiendo la notación de Alexy, simbolizaremos el peso abstracto del primer principio como GPiA y del segundo principio como GPjA.

${ }^{11}$ Cfr. Joseph Raz, Value, Respect and Attachtment, Cambridge University Press, 2001, Capítulo IV. Tiene traducción al castellano de Marta Bergas Ferriol, en prensa en la Universidad Externado de Colombia, Bogotá.

${ }^{12}$ Cfr. Con un análisis de la jurisprudencia constitucional española es estos aspectos: Carlos Bernal Pulido, El principio de proporcionalidad y los derechos fundamentales, op. cit., pp. 770 y 772 . 
la hija al hospital- proyecta sobre los principios relevantes ${ }^{13}$. La existencia de esta variable surge del reconocimiento, de que las apreciaciones empíricas relativas a la afectación de los principios en colisión pueden tener un distinto grado de certeza, y, dependiendo de ello, mayor o menor deberá ser el peso que se reconozca al respectivo principio. Así, por ejemplo, la afectación del derecho a la salud y a la vida de la hija de los evangélicos deberá considerarse como intensa, si existe certeza de que morirá de no ser ingresada en el hospital. Esta afectación, en cambio, será de menor intensidad, si los médicos no pueden identificar el problema que la aqueja, o no pueden establecer cuáles serían las consecuencias en caso de que no recibiera un tratamiento médico.

A partir de lo anterior, la pregunta es: ¿cómo se relacionan los pesos concretos y abstractos de los principios que concurren a la ponderación, más la seguridad de las premisas empíricas, para determinar, en el tercer paso, si la importancia de la satisfacción del principio contrario justifica la afectación o la no satisfacción del otro? De acuerdo con Alexy, esto es posible mediante la llamada "fórmula del peso".

\section{La fórmula del peso}

Esta fórmula tiene la siguiente estructura ${ }^{14}$ :

$$
\mathrm{GPi}, \mathrm{jC}=\frac{\mathrm{IPiC} \cdot \mathrm{GPiA} \cdot \mathrm{SPiC}}{\mathrm{WPjC} \cdot \mathrm{GPjA} \cdot \mathrm{SPjC}}
$$

Esta fórmula expresa que el peso del principio Pi en relación con el principio $\mathrm{Pj}$, en las circunstancias del caso concreto, resulta del cuociente entre el producto de la afectación del principio Pi en concreto, su peso abstracto y la seguridad de las premisas empíricas relativas a su afectación, por una parte, y el producto de la afectación del principio $\mathrm{Pj}$ en concreto, su peso abstracto y la seguridad de las premisas empíricas relativas a su afectación, por otra. Alexy mantiene que a las variables referidas a la afectación de los principios y al peso abstracto, se les puede atribuir un valor numérico, de acuerdo con los tres grados de la escala triádica, de la siguiente manera: leve $2^{\circ}$, o sea 1 ; medio $2^{1}$, o sea 2 ; e intenso $2^{2}$, es decir $4^{15}$. En cambio, a

${ }^{13}$ Cfr. Robert Alexy, "Epílogo...”, op. cit., pp. 56, especialmente la nota de pie 101. Siguiendo la notación de Alexy, denotaremos aquí la seguridad de las apreciaciones empíricas relativas a la afectación del primer principio como $S P i C$ y del segundo como $S P j C$.

${ }^{14}$ Cfr. En castellano: Ibídem. Con mayor profundidad: Robert Alexy, "Die Gewichtsformel”, en Joachim Jickeli, Meter Kreutz y Dieter Reuter Edts., Gedächtnisschrift für Jürgen Sonnenschein, De Gruyter, Berlín, 2003, pp. 771 y ss.

${ }^{15}$ Cfr. Robert Alexy, "Epílogo...”, op. cit., pp. 42 y ss. 
las variables relativas a la seguridad de las premisas fácticas se les puede atribuir un valor de seguro $2^{\circ}$, o sea, 1 ; plausible $2{ }^{\top}$, o sea $1 / 2$; y no evidentemente falso $2^{\overline{2}}$, es decir, $1 / 4$. De este modo, por ejemplo, el peso del derecho a la vida y la salud de la hija de los evangélicos podría establecerse de la siguiente manera, bajo el presupuesto de que la afectación de estos derechos se catalogue como intensa ( $\mathrm{IPiC}=4)$, al igual que su peso abstracto ( se trata de la vida!) $(\mathrm{GPiA}=4)$ y la certeza de las premisas (existe un riesgo inminente de muerte) $(\mathrm{SPiC}=1)$. Paralelamente, la satisfacción de la libertad de cultos y del derecho al libre desarrollo de la personalidad de los padres puede catalogarse como media $(\mathrm{WPjC}=2)$, su peso abstracto como medio (la religión no es de vida o muerte, podría argumentarse) (GPjA = 2) y la seguridad de las premisas sobre su afectación como intensa (pues es seguro que ordenarles llevar a la hija al hospital supone una restricción de la libertad de cultos) $(\mathrm{SPjC}=1)^{16}$.

En el ejemplo, entonces, la aplicación de la fórmula del peso al derecho a la vida y a la salud de la niña arrojaría los siguientes resultados:

$$
\mathrm{GPi}, \mathrm{jC}=\frac{4 \cdot 4 \cdot 1}{2 \cdot 2 \cdot 1}=\frac{16}{4}=4
$$

De forma correlativa, el peso de la libertad de cultos y del derecho al libre desarrollo de la personalidad de los padres sería el siguiente:

$$
\mathrm{GPj}, \mathrm{iC}=\frac{2 \cdot 2 \cdot 1}{4 \cdot 4 \cdot 1}=\frac{4}{16}=0.25
$$

Así llegaría entonces a establecerse que la satisfacción de la libertad de cultos y del derecho al libre desarrollo de la personalidad de los padres -satisfechos sólo en 0.25- no justifica la intervención en los derechos a la vida y la salud de la niña -afectados en 4-. Estos últimos derechos tendrían que preceder en la ponderación y, como resultado del caso, debería establecerse que está ordenado por los derechos fundamentales que los padres ingresen a la niña al hospital.

\section{Las cargas de argumentación}

El tercer elemento de la estructura de la ponderación son las cargas de la argumentación ${ }^{17}$. Las cargas de la argumentación operan cuando existe un

${ }^{16}$ Cfr. Ibídem, pp. 56. Asimismo, Robert Alexy, "Die Gewichtsformel...”, op. cit., pp. 789 y ss.

${ }^{17}$ Cfr. Con mayor profundidad sobre este elemento: Carlos Bernal Pulido, El principio de proporcionalidad..., op. cit., pp. 789 y ss. 
empate entre los valores que resultan de la aplicación de la fórmula del peso, es decir, cuando los pesos de los principios son idénticos ( $\mathrm{GPi}, \mathrm{jC}=\mathrm{GPj}, \mathrm{iC})$. En este aspecto, sin embargo, Robert Alexy parece defender dos posiciones, una en el capítulo final de la Teoría de los derechos fundamentales, y otra en el epílogo a dicha teoría, escrito quince años después, que podrían resultar incompatibles entre sí en algunos casos.

En la Teoría de los derechos fundamentales, Alexy defiende la existencia de una carga argumentativa a favor de la libertad jurídica y la igualdad jurídica, que coincidiría con la máxima "in dubio pro libertate"18. De acuerdo con esta carga de argumentación, ningún principio opuesto a la libertad jurídica o a la igualdad jurídica podría prevalecer sobre ellas, a menos que se adujesen a su favor "razones más fuertes" $"$. Esto podría interpretarse en el sentido de que, en caso de empate, es decir, cuando los principios opuestos a la libertad jurídica o a la igualdad jurídica no tuviesen un peso mayor sino igual, la precedencia debería concederse a estas últimas. Dicho de otra manera, el empate jugaría a favor de la libertad y de la igualdad jurídica. Como consecuencia, si una medida afectara a la libertad o a la igualdad jurídica y los principios que la respaldan no tuviesen un mayor peso que éstas, entonces, la medida resultaría ser desproporcionada y, si se tratase de una ley, ésta debería ser declarada inconstitucional.

No obstante, en el epílogo a la Teoría de los derechos fundamentales, Alexy se inclina a favor de una carga de argumentación diferente. En los casos de empate, sostiene, la decisión que se enjuicia aparece como "no desproporcionada" y, por tanto, debe ser declarada constitucional. Esto quiere decir, que los empates jugarían a favor del acto que se enjuicia, acto que en el control de constitucionalidad de las leyes es precisamente la ley. En otros términos, de acuerdo con el Alexy del epílogo, los empates no jugarían a favor de la libertad y la igualdad jurídica, sino a favor del legislador y del principio democrático en que se funda la competencia del Parlamento. De este modo, cuando existiera un empate, la ley debería declararse constitucional, por haberse producido dentro del margen de acción que la Constitución depara al legislador ${ }^{20}$.

Desde luego, la contradicción entre estas dos posturas acerca de la carga de argumentación, únicamente se presentaría cuando existiera una colisión entre la libertad jurídica o la igualdad jurídica, de un lado, y otro principio diferente a ellas, del otro. En este caso, podrían aventurarse dos interpretaciones sobre la posición de Alexy, dado que este autor no se pronuncia ex-

\footnotetext{
${ }^{18}$ Robert Alexy, Teoría de los derechos fundamentales, op. cit., pp. 549 y ss.

${ }^{19}$ Ibídem, pp. 550.

${ }^{20}$ Cfr. Robert Alexy, "Epílogo...”, op. cit., pp. 44 y ss.
} 
plícitamente acerca de esta posible contradicción. Por una parte, que Alexy cambió de postura y que, quince años después, ha revaluado su inclinación liberal y ahora privilegia al principio democrático. O, por el contrario, que Alexy persiste en conceder la carga de argumentación a favor de la libertad jurídica y la igualdad jurídica, y entonces, que en principio los empates juegan a favor de lo determinado por el Legislador, a menos que se trate de intervenciones en la libertad jurídica o la igualdad jurídica. En este caso excepcional, los empates favorecerían a estos principios.

\section{Los límites de la ponderación}

Debe señalarse que esta contradicción entre cargas de la argumentación no es el único límite de racionalidad que tiene la ponderación, por lo menos cuando se entiende con la estructura que la presenta Robert Alexy. Aquí nos referiremos a los límites que se encuentran en la ley de ponderación y en las cargas de la argumentación.

\section{Los límites racionales de la ley de ponderación}

Sobre este primer aspecto, conviene señalar que no existe un criterio objetivo para determinar los factores determinantes del peso que tienen los principios en la ley de ponderación, y que conforman la fórmula del peso, es decir: el grado de afectación de los principios en el caso concreto, su peso abstracto y la seguridad de las premisas empíricas relativas a la afectación ${ }^{21}$.

En cuanto a lo primero, es bien cierto que, como argumenta Alexy en el epílogo a la Teoría de los derechos fundamentales, en ocasiones "es posible hacer juicios racionales" ${ }^{\prime 22}$ sobre el grado en que están afectados los principios que colisionan en el caso concreto. En este sentido, existen casos fáciles en lo concerniente a la graduación de las afectaciones de los principios. Así, por ejemplo, que una revista satírica llame "tullido" a un parapléjico, constituye claramente una ofensa grave contra su derecho al honor, que, a la vez, contribuye sólo de manera leve -si es que lo hace de algún modo- a la satisfacción de la libertad de información.

Sin embargo, junto a estos casos fáciles existen siempre casos difíciles, en los que las premisas que fundamentan la graduación, y no sólo las fácticas sino también las analíticas y las normativas, son extremadamente inciertas. Así tiende a ocurrir, por ejemplo, en todos los casos en los que está en juego la libertad religiosa. De ordinario, la gravedad de una intervención en

\footnotetext{
${ }^{21} \mathrm{Cfr}$. Sobre algunas reglas argumentativas para determinar la magnitud de estos factores: Carlos Bernal Pulido, El principio de proporcionalidad..., op. cit., pp. 760 y ss.

${ }^{22}$ Cfr. Robert Alexy, "Epílogo...”, op. cit., pp. 33 y ss.
} 
la libertad religiosa no es susceptible de determinarse en abstracto, con base en criterios objetivos o, si se quiere, intersubjetivos, sino que, por el contrario, es algo que en principio sólo podría establecer el creyente involucrado y que dependería de su subjetividad. La gravedad de obligar a un evangélico a llevar a su hija al hospital o a un testigo de Jehová a autorizar la práctica de una transfusión de sangre para su hijo o para sí mismo, es algo que sólo el titular de la libertad religiosa puede precisar. Para un creyente puede ser más importante la muerte bajo el cumplimiento de sus reglas religiosas, que la continuación de una vida impura, en pecado, a la que sobrevenga una condena eterna. En general, esta modalidad de casos difíciles se presenta cuando lo que está en juego en la ponderación es un margen de libertad o de autonomía que la Constitución ha deparado a un individuo o a un colectivo. En este sentido, se presenta el mismo fenómeno, cuando los objetos que concurren a la ponderación son un derecho fundamental -la integridad física, verbigracia- y la autonomía de una comunidad. De este fenómeno es ejemplo, el caso en que, de acuerdo con sus leyes tradicionales, cuya aplicación está avalada por la Constitución, las autoridades de una comunidad indígena colombiana imponen a un infractor un pena consistente en 60 latigazos $^{23}$. Es probable que, desde la perspectiva de la sociedad mayoritaria, los latigazos se consideren casi unánimemente como una afectación grave del derecho a la integridad física. No obstante, desde esta perspectiva será muy difícil catalogar atinadamente el grado de afectación de la autonomía de la comunidad indígena, que llevaría consigo la inaplicación de la ley tradicional que ordena los latigazos. Así como cuando está en juego la libertad religiosa, no está claro cuál es el punto de vista a partir del cual debe hacerse la graduación. Y esta duda sólo puede ser resuelta por el operador jurídico -el juez sobre todo-, después de adoptar una postura material e ideológica. Un juez más respetuoso de la libertad religiosa o de la autonomía de las comunidades indígenas, hará valer el punto de vista interno del afectado. Por el contrario, un juez más partidario de la universalidad de los derechos humanos y de la imposición de los valores de la sociedad mayoritaria, hará prevalecer la visión de esta última. Así las cosas, este aspecto de la ponderación depararía al juez un margen de acción, en el que éste puede hacer valer su ideología política ${ }^{24}$ para encaminarse, en términos de Duncan Kennedy, a "la-sentencia-a-la-que-quiere-llegar"25.

${ }^{23}$ El caso es de la Sentencia T-523 de 1997.

${ }^{24}$ Cfr. Duncan Kennedy, A Critique of Adjudication (fin de siècle), Harvard University Press, Cambridge - Londres, 1997, pp. 1.

${ }^{25}$ Cfr. Duncan Kennedy, Libertad y restricción en la decisión judicial, Traducción de Diego López Medina y Juan Manuel Pombo, Ediciones Uniandes, Bogotá, 1999, pp. 91 y ss. 
Además de lo anterior, también la ponderación depara un margen de acción al intérprete, cuando existen dudas sobre si un caso es fácil o difícil en cuanto a la graduación de la afectación de los principios. Puede suceder que incluso un caso que parece fácil, resulte ser en realidad un caso difícil. Esto puede mostrarse con un ejemplo al que alude el propio Robert Alexy y que se refiere a la sentencia sobre el tabaco del Tribunal Constitucional Alemán ${ }^{26}$. Alexy considera que esta sentencia es representativa del conjunto de los "ejemplos fáciles en los que resulta plausible formular juicios racionales sobre las intensidades de las intervenciones en los derechos fundamentales y sobre los grados de realización de los principios, de tal modo que mediante la ponderación pueda establecerse un resultado de forma racional." La sentencia versa sobre el deber de los productores de tabaco de colocar etiquetas que adviertan del peligro para la salud que implica fumar. Alexy sostiene que ésta es una intervención "relativamente leve en la libertad de profesión y oficio" 27 , sobre todo si se le compara con otras medidas alternativas: la prohibición de expender tabaco o la restricción en su venta. Correlativamente, Alexy piensa que esta medida satisface el principio contrapuesto, la protección de la salud, de manera intensa o alta. Como argumento señala: "El Tribunal Constitucional no debía de exagerar, cuando, en su Sentencia sobre las advertencias acerca del tabaco, considera cierto, "de acuerdo con el estado de los conocimientos de la medicina actual", que fumar origina cáncer, así como enfermedades cardiovasculares" ${ }^{" 28}$. De este modo, la afectación leve de la libertad de profesión y oficio se enfrentaría a una satisfacción intensa del derecho a la salud.

Ahora bien, cabe reconocer que esta argumentación de Alexy frente al caso no es la única viable. Por el contrario, existen graduaciones alternativas, que podrían llevar a soluciones diferentes. Aquí sobre todo podría tenerse en cuenta que, desde el punto de vista fáctico, es bien discutible que la obligación de etiquetar las cajetillas de cigarrillos con advertencias sobre los riesgos que fumar ocasiona para la salud, pueda implicar una satisfacción intensa del derecho a la salud. Bien puede pensarse que la eficacia disuasoria de estas etiquetas es mínima o inclusive nula, porque la información que divulga es altamente conocida; porque la adicción al tabaco no es el resultado de la carencia de información sobre su carácter nocivo, sino más bien un caso claro de debilidad de la voluntad; e incluso - un argumento irónico- porque en ocasiones para la mente humana lo prohibido y lo nocivo es lo más apetecido. Si se observan las cosas desde esta perspectiva, enton-

\footnotetext{
${ }^{26}$ Cfr. BVerfGE 95, 173 (184).

${ }^{27}$ Cfr. Robert Alexy, “Epílogo...”, op. cit., pp. 33.

${ }^{28}$ Ibídem.
} 
ces, en lo concerniente al grado en que se satisface el derecho a la salud, puede concluirse que la graduación que Alexy -y el Tribunal Constitucional alemán- lleva a cabo, está errada, o que, en este punto, se trata de un caso difícil.

Ahora bien, esta dificultad para determinar el punto de vista correcto para la graduación de la afectación de los principios y los argumentos correctos en los casos difíciles, también se presenta en lo referente a la fijación del peso abstracto y de la seguridad de las premisas relevantes en la ponderación.

El peso abstracto es una variable muy singular, que remite siempre a consideraciones ideológicas y hace necesaria una toma de postura por parte del intérprete sobre aspectos materiales, relativos a la idea de Constitución, de Estado y de Justicia. Naturalmente, la variable del peso abstracto pierde toda su importancia, cuando los principios enfrentados en la ponderación son de la misma índole. Los pesos abstractos se anulan, cuando, por ejemplo, se establece una colisión entre un mismo derecho fundamental ejercido por dos titulares diversos - dos grupos políticos contrarios que quieren manifestarse en la misma calle de una ciudad a la misma hora y en el mismo día y es posible que la manifestación simultánea derive en peleas entre los grupos-. Sin embargo, muy por el contrario, los pesos abstractos adquieren gran relieve, cuando en la colisión confluyen derechos o principios distintos, y presentan características que lleven a atribuirles un peso abstracto mayor o menor. De este modo, es posible otorgar un peso abstracto mayor al derecho a la vida o a los derechos fundamentales que tienen una conexión con el principio democrático - la libertad de información, verbigracia- o con la dignidad humana ${ }^{29}$-el derecho a la intimidad o a la integridad física-, o simplemente, cuando la propia Constitución lo establece de alguna manera, como cuando el artículo 44 del texto colombiano prescribe que "Los derechos de los niños prevalecen sobre los derechos de los demás". Correlativamente, también puede otorgarse un peso abstracto menor a los principios que colisionan con los derechos fundamentales y que no aparecen en la Constitución, sino que han sido establecidos por el Legislador dentro de su margen para la determinación de fines y están respaldados en última instancia por el principio democrático.

A pesar de todo lo anterior, es necesario reconocer que la fijación del peso abstracto también tiene ciertos límites de racionalidad, que asimismo deparan un espacio a la subjetividad del intérprete. Bien difícil resulta esta-

${ }^{29}$ Cfr. Sobre el principio democrático y la dignidad humana como elementos relevantes para la fijación de peso abstracto de los principios, las sentencias colombianas: Sentencias T556 de 1998 y T-796 de 1998. 
blecer una completa graduación preestablecida de pesos abstractos, que se formule en términos de la escala triádica. Es posible que la idea de que el derecho a la vida tenga el valor más elevado (4) no concite ningún desacuerdo. Pero, a partir de allí, ¿cuál es el valor que debe otorgarse a los derechos que están vinculados con el principio democrático o con la dignidad humana? Y, además, ¿ese valor debe ser igual para todos los derechos, o puede cambiar de acuerdo con lo estrecho o laxo del nexo que esos derechos tengan con dichos principios? ¿Tendría entonces la libertad de información el mismo peso abstracto que la vida (4), o debe estimarse que tiene sólo un peso abstracto medio (2)? Estas dificultades surgen, porque la graduación del peso abstracto en el marco de la escala triádica, pasa por el mismo problema que presenta la construcción del llamado por Böckenförde "orden fundamental", en el que la Constitución aparece como una detallada escala jerárquica de todos los derechos y principios existentes. Aunque la graduación del peso abstracto es menos compleja, porque no exige la construcción de una detallada jerarquía ordinal, sino sólo la clasificación de los principios en tres rangos de peso, en esta operación no deja de ser fundamental la influencia de la ideología del intérprete. De este modo, un juez más individualista, otorgará a la libertad general de acción y a las libertades específicas el peso abstracto más alto y a los principios que tengan que ver con la colectividad un peso menor. Lo contrario hará un juez que actúe bajo el prurito de lograr la construcción, la integración y la defensa de la comunidad.

Por último, los límites de racionalidad también aparecen al intentar establecer la certeza de las premisas empíricas relativas a la afectación de los principios. Como hemos expuesto en otro lugar ${ }^{30}$, desde el punto de vista empírico, la afectación de un principio depende de la mayor o menor eficacia, rapidez, probabilidad, alcance y duración de la intervención que en él implique la medida enjuiciada en la ponderación. De esta manera, la afectación negativa y la satisfacción de los principios será mayor, cuanta mayor eficacia, rapidez, probabilidad, alcance y duración ostente la medida examinada. En este punto las posibilidades de racionalidad están limitadas, en primer lugar, en razón de la dificultad para establecer la certeza de las premisas empíricas desde todas esas perspectivas, esto a su vez, porque los conocimientos empíricos del intérprete también son limitados. Al mismo tiempo, y en segundo lugar, las limitaciones surgen de la complejidad que resulta al combinar las variables. ¿Cómo debe catalogarse, por ejemplo, la certeza de una premisa empírica cuya eficacia puede establecerse de forma plausible $(1 / 2)$, su rapidez de manera no evidentemente falsa $(1 / 4)$, su proba-

\footnotetext{
${ }^{30}$ Carlos Bernal Pulido, El principio de proporcionalidad y los derechos fundamentales, op. cit., pp. 763 y ss.
} 
bilidad segura (1), su alcance plausible (1/2) y su duración segura (1)? Y, correlativamente, ¿será mayor esa certeza si a las mismas variables se les atribuyen los mismos valores de seguridad pero en un orden distinto: eficacia $(1 / 4)$, rapidez (1), probabilidad ( $1 / 2)$, alcance (1) y duración ( $1 / 2)$ ? En fin ¿cuál de estas variables es más determinante de la certeza, en definitiva?

A partir de ello sólo puede concluirse que sobre este aspecto el intérprete también dispone de un margen irreducible de subjetividad, en el que puede hacer valer sus apreciaciones empíricas sobre las circunstancias en que se desarrolla la ponderación.

\section{Los límites de racionalidad en las cargas de la argumentación}

Como antes observamos, la contradicción entre las cargas de argumentación in dubio pro libertate e in dubio pro legislatore también constituye un límite a la racionalidad de la ponderación, que depara al intérprete un margen de subjetividad. La aplicación de una u otra carga depende de la postura ideológica del juez. Un juez que quiera dar prevalencia al principio democrático, operará siempre con el in dubio pro legislatore y, de este modo, concederá al Parlamento la posibilidad de equilibrar los principios en conflicto mediante un empate entre sus pesos específicos. Por el contrario, un juez liberal se servirá en todo caso del in dubio pro libertate y declarará desproporcionadas a aquellas medidas que no consigan favorecer al principio que constituye su finalidad, en un grado mayor a aquél en que se afecta la igualdad jurídica o la libertad jurídica. Esta igualdad y esta libertad, aducirá, son los pilares del Estado de Derecho y su sacrificio sólo se justifica cuando se obtienen beneficios mayores. Finalmente, es posible que el juez defienda soluciones matizadas que combinen la aplicación de una $\mathrm{u}$ otra carga argumentativa o que sea el resultado de una ponderación entre ellas. Así, entonces, podría aplicarse el in dubio pro legislatore para las medidas ordinarias de afectación de los derechos fundamentales y reservar el in dubio pro libertate para las medidas que en el caso concreto afecten intensamente a la igualdad jurídica o a la libertad jurídica. O, también, se podría considerar la aplicación del in dubio pro libertate como la regla general y destinar el in dubio pro legislatore a áreas que las que el Parlamento tiene un margen de acción más amplio en razón de la materia, como la política económica o la política criminal. No parece desatinado sostener que una Constitución abierta permitiría cualquiera de estas posibilidades, porque contiene, al mismo tiempo, los principios, a veces contrarios entre sí, de la democracia y la libertad, de la igualdad jurídica y la igualdad fáctica, de la construcción de la comunidad y el respeto a la órbita individual. 


\section{Conclusión}

Todo lo anterior muestra que la ponderación no es un procedimiento algorítmico que por si mismo garantice la obtención de una única respuesta correcta en todos los casos. Por el contrario, tiene diversos límites de racionalidad que deparan al intérprete un irreducible margen de acción, en el que puede hacer valer su ideología y sus propias valoraciones. Sin embargo, el hecho de que la racionalidad que ofrece la ponderación tenga límites, no le enajena su valor metodológico, así como la circunstancia de que el silogismo no garantice la verdad de las premisas mayor y menor, tampoco le resta por completo su utilidad. La ponderación representa un procedimiento claro, incluso respecto de sus propios límites. Si bien no puede reducir la subjetividad del intérprete, en ella sí puede fijarse, cuál es el espacio en donde yace esta subjetividad, cuál es el margen para las valoraciones del juez y cómo dichas valoraciones constituyen también un elemento para fundamentar las decisiones. La ponderación se rige por ciertas reglas que admiten una aplicación racional, pero que de ninguna manera pueden reducir la influencia de la subjetividad del juez en la decisión y su fundamentación. La graduación de la afectación de los principios, la determinación de su peso abstracto y de la certeza de las premisas empíricas y la elección de la carga de la argumentación apropiada para el caso, conforman el campo en el que se mueve dicha subjetividad.

Bogotá, 30 de julio de 2003 